\title{
Experimental and Numerical Investigation on the Flow-Induced Interior Noise Based on Pellicular Analysis
}

\author{
Pan Zuofeng and Hou Hangsheng \\ China Faw Group Co. Ltd, State Key Laboratory of Comprehensive Technology on Automobile Vibration and \\ Noise \& Safety control, Changchun 13000, China.
}

Lu Wenbo and Ji Changwei

Beijing Hi-key Technology Co., Ltd., Beijing 100192, China.

Du Mintao and Yin Xiang

School of automotive engineering, Wuhan University of Technology, Wuhan 430070, China.

\author{
Bai Changan \\ Hexagon - Free Field Technologies Acoustics team, Shanghai 20030, China.
}

(Received 25 December 2019; accepted 9 November 2020)

Turbulence caused by the rearview mirror and A-pillar is the main source of interior wind noise. Because of the difference in energy and transfer efficiency of acoustic and turbulent pressure, it is important to accurately identify the two kinds of pressure on the window surface when studying interior wind noise. Wave Number Decomposition (WND) and Pellicular Mode Decomposition (PMD) are successfully applied to decompose the pressure on the window. Furthermore, the transfer function calculated by pellicular analysis is used to calculate the interior wind noise, and the result is compared with the result calculated by the Finite Element Method (FEM) and measured by the experiment. The results revealed that the interior wind noise calculated by the pellicular analysis method is almost the same as the result calculated by the FEM, and that it presents obvious advantages in computational efficiency.

\section{INTRODUCTION}

With the improvement of people's consumption level and concept, the NVH (noise, vibration, and harshness) performance of vehicle has gradually become one of hot points. Wind noise generated by the A-pillar and side mirror is the main source of the interior noise when a car is travelling at high speed. Due to the high cost of wind tunnel experiments, numerical simulation has become the main method to study vehicle wind noise.

Previous research shows that when a car runs at high speed, turbulent and acoustic pressure acting on the side windows will cause their vibration, and is the main source of interior wind noise. ${ }^{1,2}$ Although the energy of acoustic pressure is much smaller than that of turbulence pressure, the transfer efficiency is the opposite. Moreover, the interior noise generated by the two pressure is correlated with specific model. ${ }^{3}$ Therefore, when studying the interior noise caused by the external flow field, turbulence and acoustic pressure should be considered at the same time. Accurate separation and identification of the two pressures is very important for taking corresponding noise reduction measures in a specific frequency band. At present, several scholars have carried out some research in distinguishing the two pressures by Wave Number Decomposition (WND) ${ }^{4-6}$ However, WND can only decompose the pressure on a regular rectangular surface. The side windows in vehicle are not a regular rectangle.

Pellicular Mode Decomposition (PMD) is a computation procedure that is mainly used to compute the radiation of a vibrating system. Since the pellicular modes form an orthogonal basis, it is convenient to compute the solution of various acoustic problems (sound radiation, inverse problem) using a related basis. PMD can be used to decompose the pressure on irregular surfaces. ${ }^{7,8}$ However, using PMD to decompose the pressure on the side windows of a real vehicle had not been carried out until now.

In terms of interior wind noise calculation, the Finite Element Method (FEM) and Statistical Energy Analysis (SEA) have been widely used. ${ }^{9-14}$ But both of these methods have the same disadvantage: if the external shape changes, all the flow field and acoustic calculations need to be restarted.

Therefore, the pellicular analysis method is applied to calculate the vehicle interior wind noise. Since the pellicular modes depend only on the geometry of the boundary surface and the acoustic material properties, the radiation database made from pellicular modes could be re-used when the external structure changes (the shape of the boundary surface remains unchanged). ${ }^{15}$ Then, the calculation time for the sound propagation could be greatly shortened. 


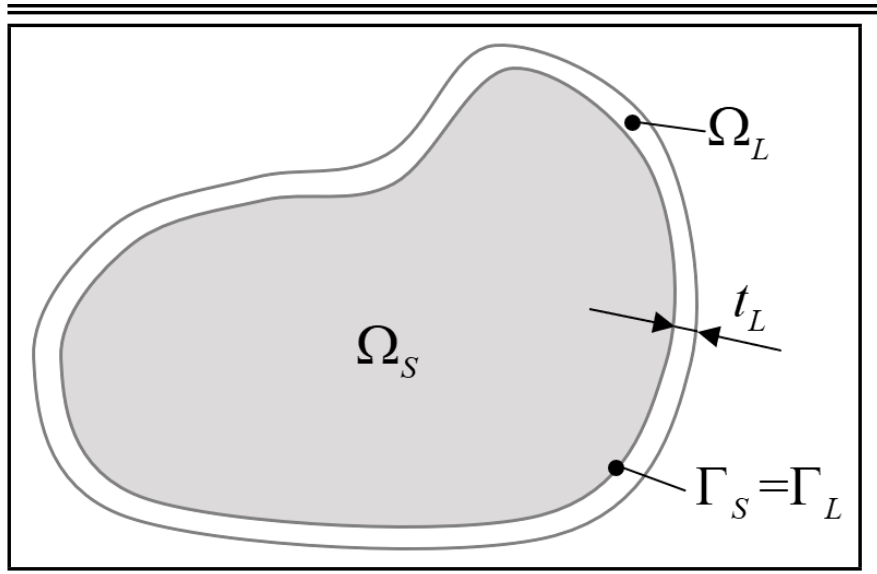

Figure 1. Pellicular acoustic domain

In this paper, the pressure on side window is first decomposed into turbulent and acoustic pressure by WND and PMD respectively, then the interior wind noise was computed by the pellicular analysis method. Meanwhile, the comparison between the calculation and experiment was carried out to confirm the computational approach.

\section{BASIC THEORY ABOUT PELLICULAR ANALYSIS}

The pellicular modes are defined as the set of acoustic modes related to pellicular domain $\Omega_{L}$ (see Fig. 1). $\Omega_{S}$ is the geometrical domain, $\Gamma_{S}$ and $\Gamma_{L}$ is the boundary of pellicular domain and geometrical domain. In a continuous framework, these modes result from the solution of the following eigenvalue problem:

$$
K_{L}\left(\Phi_{i}(x, y, z)\right)=\lambda_{i} M_{L}\left(\Phi_{i}(x, y, z)\right) ;
$$

where $K_{L}$ and $M_{L}$ are the so-called acoustic stiffness and mass operators respectively, while $\Phi_{i}(x, y, z)$ and $\lambda_{i}$ denote a particular eigenvector/eigenvalue pair.

These modes can be verified by the orthonormality conditions:

$$
\begin{aligned}
& \int_{\Gamma_{L}} \Phi_{i}(x, y, z) K_{L}\left(\Phi_{j}(x, y, z)\right) d \Gamma_{L}=\lambda_{i} \delta_{i j} \\
& \int_{\Gamma_{L}} \Phi_{i}(x, y, z) M_{L}\left(\Phi_{j}(x, y, z)\right) d \Gamma_{L}=\delta_{i j} .
\end{aligned}
$$

Any pressure field $p(x, y, z)$ and velocity field $v_{n}(x, y, z)$ along the window surface can be expanded as:

$$
\begin{aligned}
p(x, y, z) & =\sum_{i=1}^{M_{L}} \Phi_{i}(x, y, z) x_{i}^{p} \\
v_{n}(x, y, z) & =\sum_{i=1}^{M_{L}} \Phi_{i}(x, y, z) x_{i}^{v_{n}} .
\end{aligned}
$$

The participation factors $x_{i}^{p}$ and $x_{i}^{v_{n}}$ can be obtained using the orthonormality property:

$$
\begin{aligned}
x_{i}^{p} & =\int_{\Gamma_{L}} \Phi_{i}(x, y, z) p(x, y, z) d \Gamma_{L} \\
x_{i}^{v_{n}} & =\int_{\Gamma_{L}} \Phi_{i}(x, y, z) v_{n}(x, y, z) d \Gamma_{L} .
\end{aligned}
$$

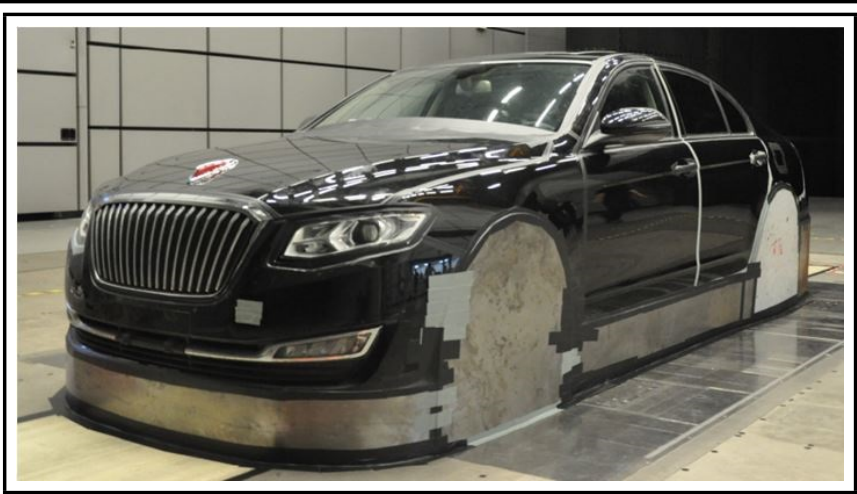

Figure 2. Test vehicle.

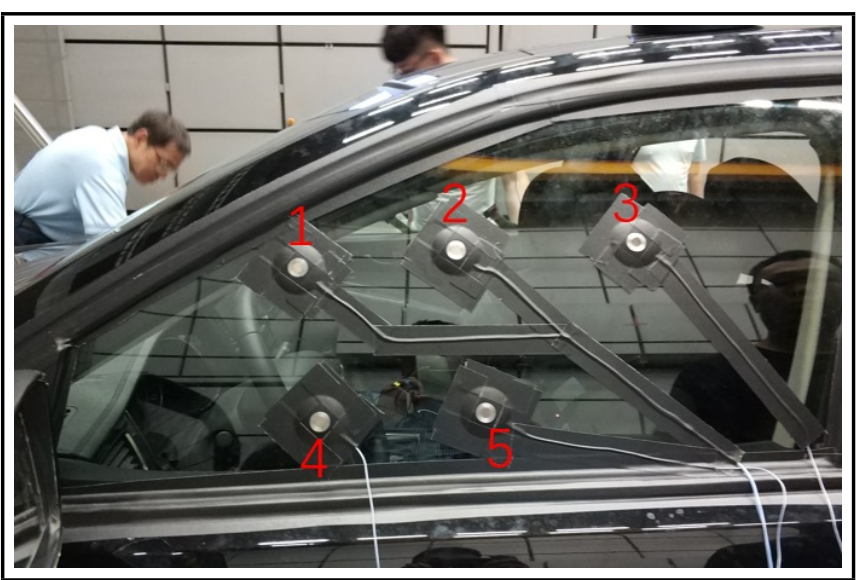

Figure 3. Microphones' position.

For a given acoustic domain, a radiation database containing the acoustic pressure $\Pi_{i j}(\omega)$ can be obtained at given field points induced by velocity boundary conditions:

$$
v_{n}(x, y, z)=\Phi_{j}(x, y, z) \quad(x, y, z) \in \Gamma_{L} .
$$

Similarly, the boundary pressure $P_{j}$ induced by the same boundary condition can be obtained:

$$
P_{j}=\sum_{i=1}^{M_{L}} \Phi_{j} \beta_{i j}
$$

where $\beta_{i j}$ is given by:

$$
\beta_{i j}=\int_{\Gamma_{L}} \Phi_{i}(x, y, z) P_{j}(x, y, z) d \Gamma_{L} .
$$

These fields depend only on the geometry of the radiator and the properties of the acoustic domain. They can be computed and stored in a radiation database for a given set of discrete frequencies. Once the transfer functions have been obtained, the interior sound pressure level (SPL) can be predicted by taking the product of the pressure participation factors and the matrices of pellicular mode transfer function:

$$
p_{j}=\sum_{i=1}^{L} \Pi_{i j}(\omega) x_{p}^{i}
$$

where $p_{j}$ is the interior pressure at location $j, \Pi_{i j}(\omega)$ is the transfer function in frequency $\omega, L$ is the number of pellicular modes considered in the pressure decomposition. 


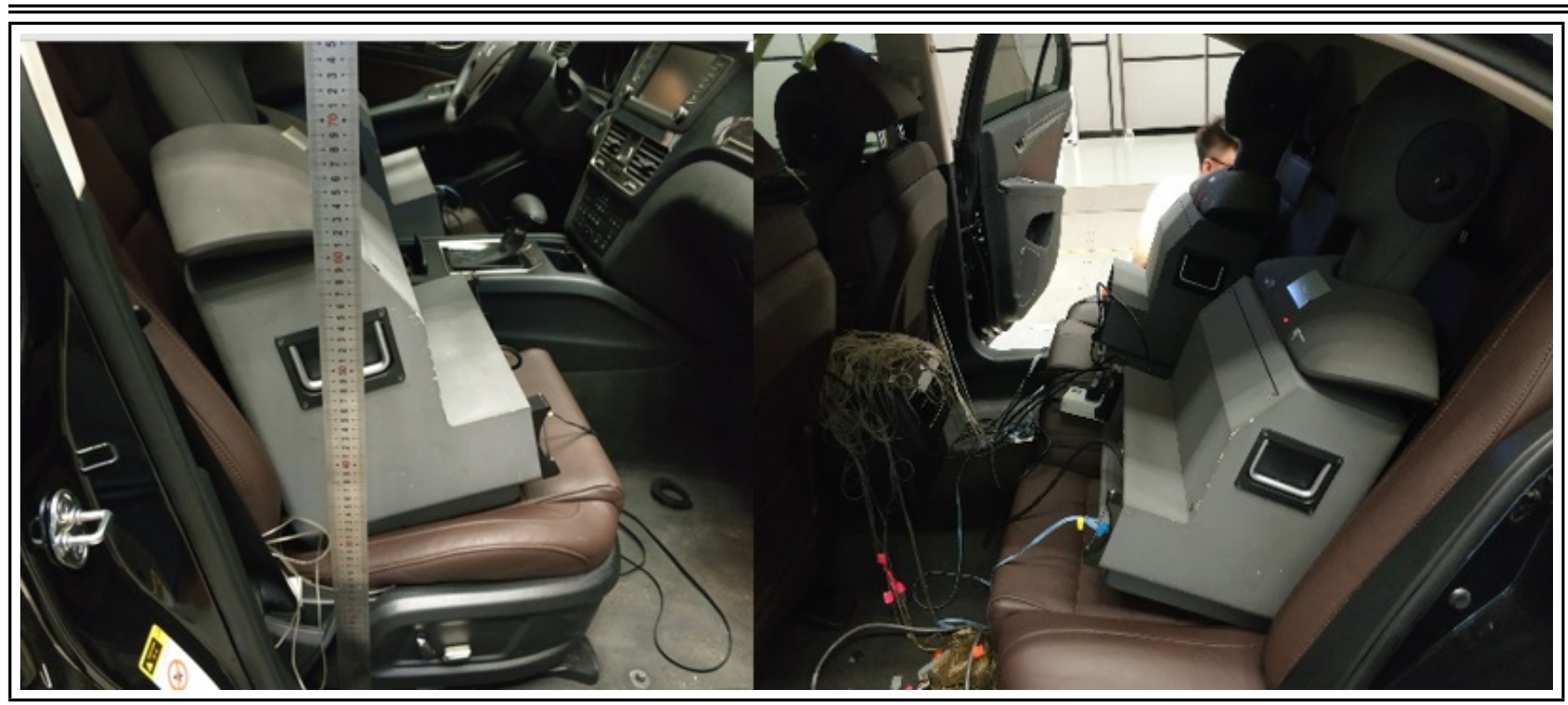

Figure 4. Dummy heads inside the vehicle.

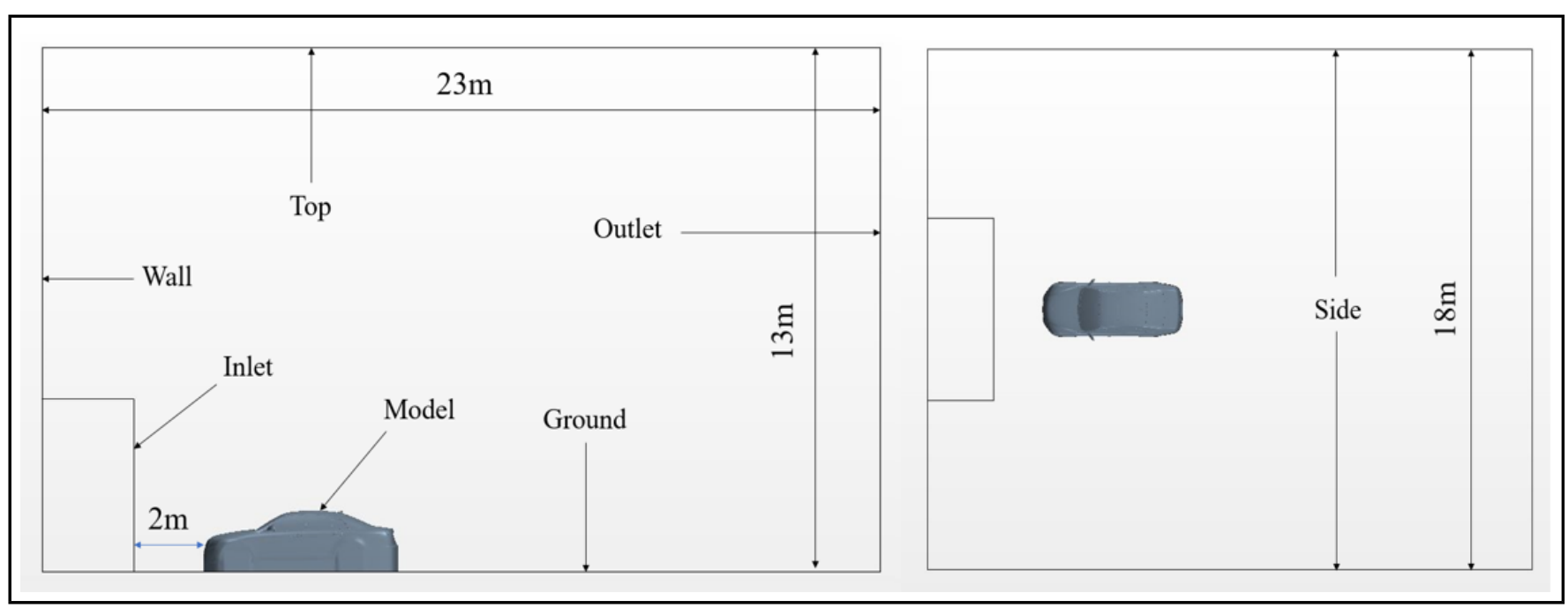

Figure 5. Calculation domain.

Table 1. Settings of the boundary condition.

\begin{tabular}{|c|c|}
\hline Boundary of the computational domain & Setting \\
\hline Inlet & Velocity inlet, 140 km/h \\
Outlet & Pressure outlet, 0 Pa \\
Model and Ground & No-slip wall \\
Other boundaries & Slip wall \\
\hline
\end{tabular}

\section{EXPERIMENTS}

The physical model, Hongqi H7, was tested at the Shanghai Ground Vehicle Wind Tunnel Center (SGVWTC). The wind tunnel at SGVWTC is an acoustic wind tunnel. The test vehicle was installed at the test section (see Fig. 2). The air-inlet grille and body gaps were sealed with tape, and chassis and wheels were partially closed.

Five microphones were installed on the surface of the left window to measure turbulence pressure fluctuation. The position of the microphones is shown in Fig. 3.

As shown in Fig. 4, four dummy heads were installed in the driver's seat, the front passenger seat, and the rear seats.

The test vehicle was in the state of 0 yaw angle and the in-

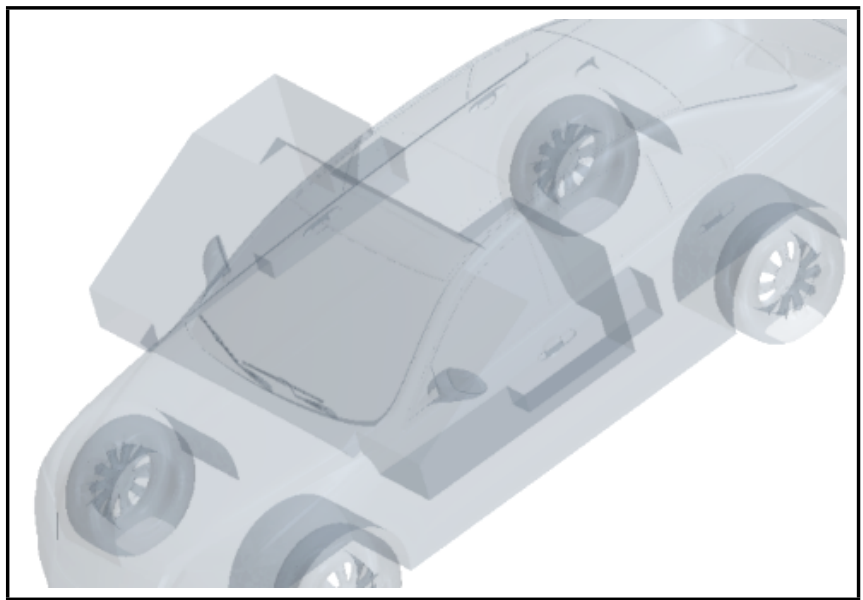

Figure 6. The sound source areas.

coming wind speed was $120 \mathrm{~km} / \mathrm{h}$. When the flow field around the vehicle reached a stable state, the microphones started to collect data. The sampling time was $10 \mathrm{~s}$, and the time step was $2.08 \times 10^{-5} \mathrm{~s}$. 


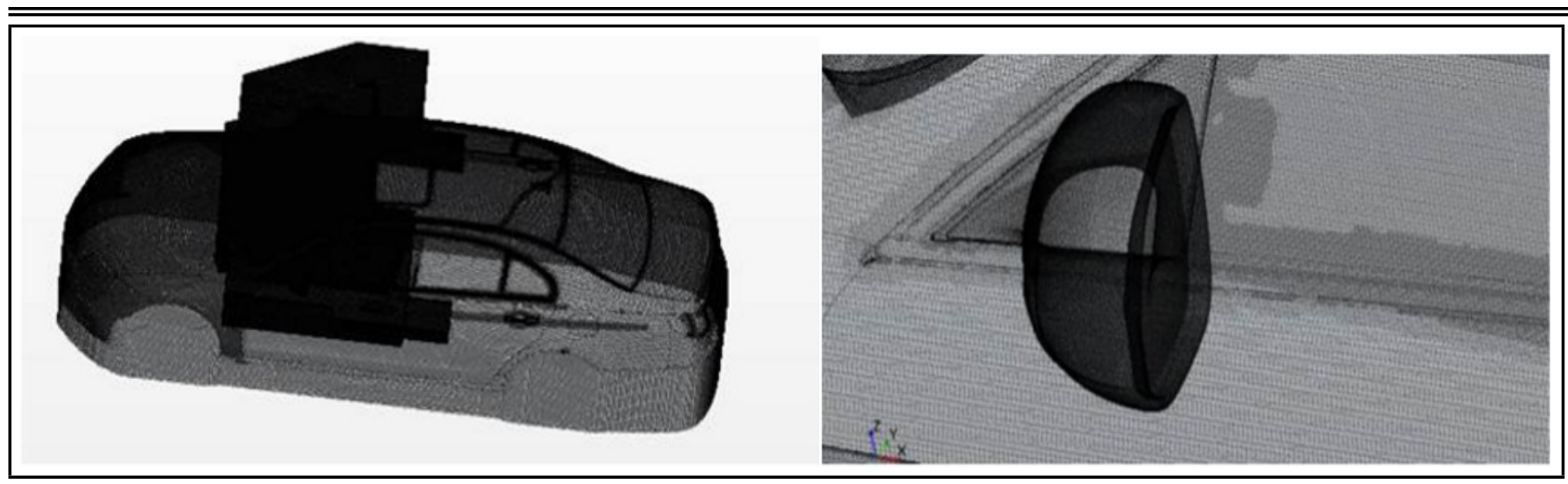

Figure 7. The surface grid of the car.

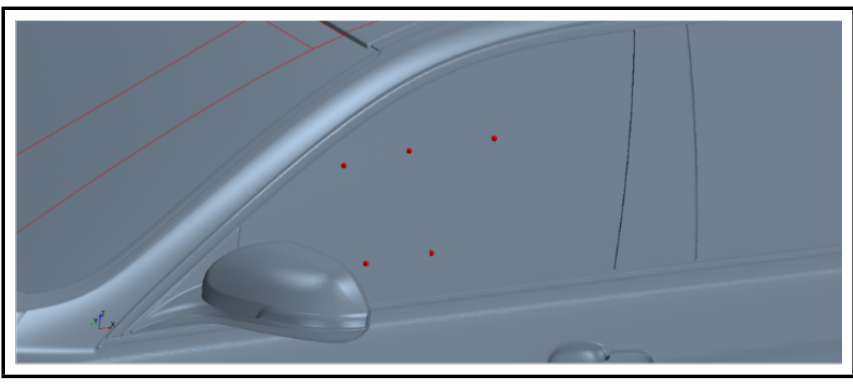

Figure 8. Pressure monitoring points.

\section{NUMERICAL SIMULATION OF THE EXTERIOR FLOW FLIED}

A computational fluid dynamic (CFD) domain, the same as the real wind tunnel dimensions was constructed, as shown in Fig. 5. The length, width and height are $23 \mathrm{~m}, 18 \mathrm{~m}$ and $13 \mathrm{~m}$, respectively. The distance between the velocity inlet and the vehicle model was $2 \mathrm{~m}$. In addition, the settings of the boundary condition for the computational domain are shown in Table 1 .

Due to the presence of protrusions such as wipers, rearview mirrors and handles, the flow field in the area near these components changed dramatically, causing the window glass to vibrate and radiate noise into the car. These areas were set to sound source areas, as shown in Fig. 6.

The surface grid size on the car was $2-10 \mathrm{~mm}$, the maximum size of volume grid in the sound source field was $4 \mathrm{~mm}$, and the maximum size of the volume grid close to the rearview mirror, pillar A and door handle was $1 \mathrm{~mm}$. These grid sizes were suitable to capture the turbulent structures exhibiting frequencies of up to $3000 \mathrm{~Hz}$. For LES, the Y+ value on the surface of the sound source areas should be about 1 . Therefore, a 5-layer boundary layer grid was set on the surface of the car, and the thickness of the first layer was $0.02 \mathrm{~mm}$, and the total thickness was $2 \mathrm{~mm}$. Finally, the total size of grid was approximately 0.15 billion. The surface grid is shown in Fig. 7.

To validate the accurateness of numerical simulation, as shown in Fig. 8, the pressure monitoring points whose positions were the same as the microphones in the experiment were set up in the left window to obtain the turbulence pressure fluctuation.

The STAR-CCM+ was used to solve the flow field. To make the transient computation converge faster, the steadystate computation was first applied to the solution. The set-

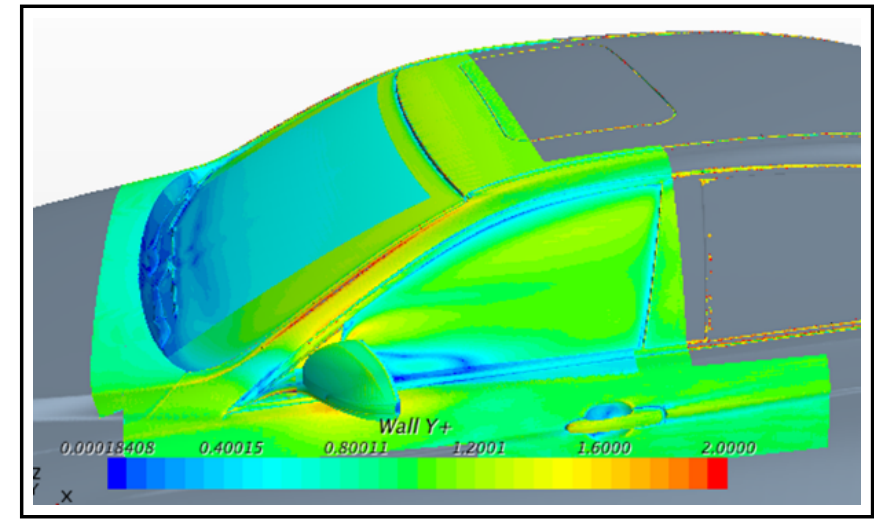

Figure 9. Y+ Value on the surface of the sound source areas.

Table 2. Settings of the steady-state solution.

\begin{tabular}{|c|c|}
\hline Setting item & Setting \\
\hline Time & Steady \\
Equation of state & Ideal gas \\
Turbulence model & k-epsilon \\
Wall function & Two-layer all Y+ wall treatment \\
\hline
\end{tabular}

Table 3. Settings of the transient solution.

\begin{tabular}{|c|c|}
\hline Setting item & Setting \\
\hline Time & Implicit unsteady \\
Equation of state & Ideal gas \\
Turbulence model & Large eddy simulation \\
Subgrid scale model & Smagorinsky model \\
Wall function & Two-layer all Y+ wall treatment \\
\hline
\end{tabular}

Table 4. Computational resources.

\begin{tabular}{|c|c|c|}
\hline & Steady & Transient \\
\hline CPU & Intel Xeon E5-2690 v4 & Intel Xeon E5-2690 v4 \\
Host & 20 & 20 \\
Memory & $128 \mathrm{~GB}$ & $128 \mathrm{~GB}$ \\
Time & 3 days & 6 days \\
\hline
\end{tabular}

tings of the steady-state solution are listed in Table 2. After the steady-state solution converged, the flow field result obtained from the steady-state computation was taken as the initial field of the transient solution. The comparison frequency between simulation and experiment was $100-3000 \mathrm{~Hz}$. The transition flow field computation was set with time step of $2 \times 10^{-5} \mathrm{~s}$, sampling time of $0.1 \mathrm{~s}$, and other settings of transient solution are listed in Table 3. The computational resources are listed in Table 4. 


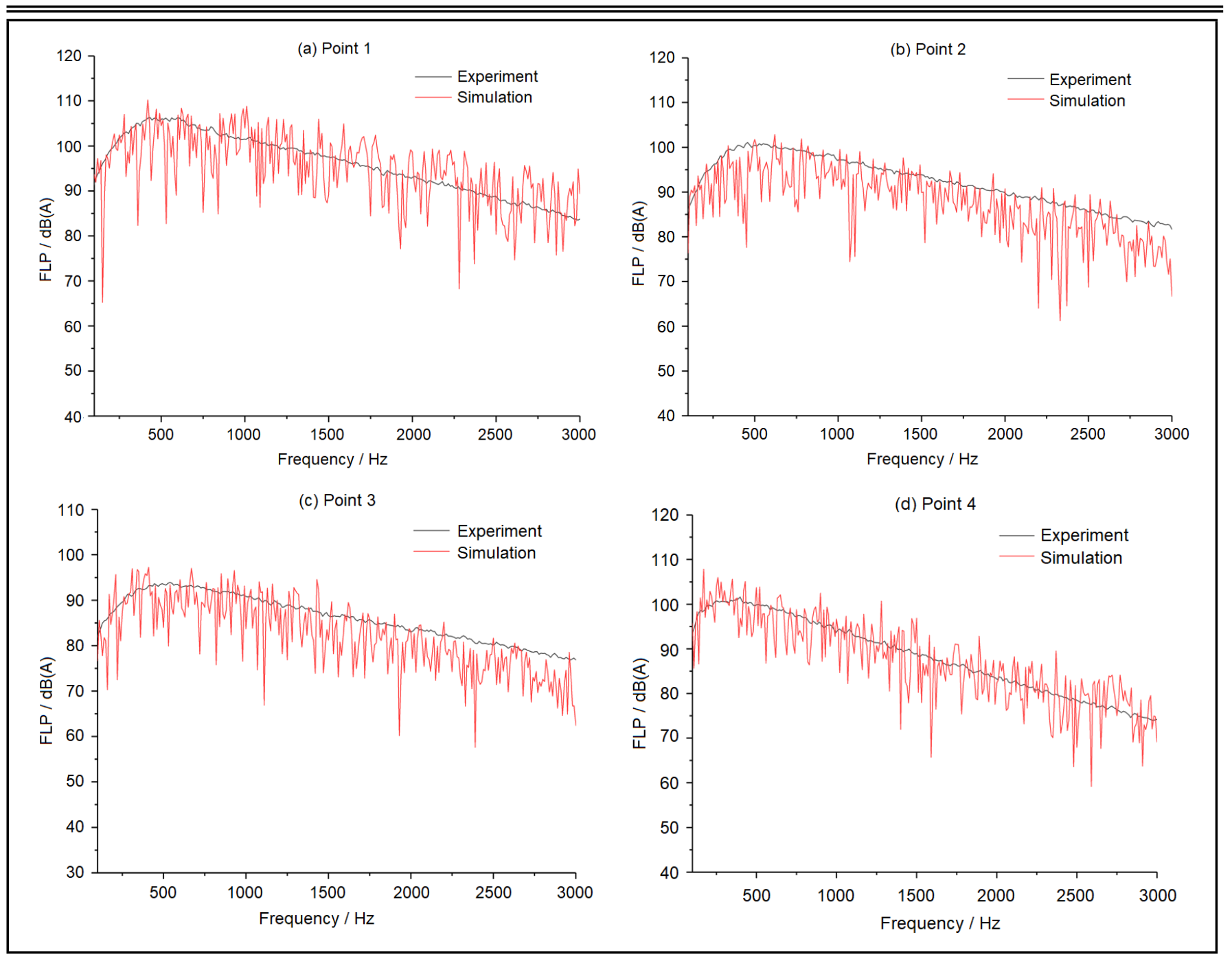

Figure 10. Comparison of the simulation and the experiment.

\section{RESULTS OF THE FLOW FIELD}

The $\mathrm{Y}+$ values on the surface of the sound source areas are illustrated in Fig. 9. The Y+ values in this region are between 0 and 2 , and the values in the side mirror are less than 1 , indicating that the near-wall grid meets the calculation requirement.

Accurate flow field results are the basis of acoustic calculation. The fluctuation pressure level (FPL) comparisons between the numerical simulation and the experiment at the monitoring points are plotted in Fig. 10. The results revealed that the numerical simulation has a good agreement with the experimental results. In other words, the flow flied computation have an enough accuracy for pressure decomposition and interior wind noise calculation.

\section{WALL PRESSURE DECOMPOSITION}

The pressure fluctuation on the surface of the window was considered to be made up of two parts: 1) Due to the compressibility of the fluid, the vortex contraction and deformation in the flow and their interaction, as well as the interaction between the vortex and the wall surface, will produce noise source. This source causes acoustic pressure. 2) When the air flows through the glass surface, it causes turbulence pressure.

As to the two pressure contributions on the glass surface, the acoustic contribution was usually above $1000 \mathrm{~Hz}$ with a relatively small amplitude (about $0.1 \mathrm{~Pa}$ ), while the turbulence contribution was usually below $1000 \mathrm{~Hz}$ with a relatively large amplitude (about 100-1000 Pa) ${ }^{8}$ Although the acoustic pressure energy was smaller than the turbulence pressure, the transfer efficiency of the two was the opposite. Therefore, the above two pressures should be considered simultaneously when studying the interior wind noise in the vehicle.

In this paper, ACTRAN was used to decompose the surface pressure. WND method was used to decompose the pressure on the sub-surface firstly, the pressure participation factored on a rectangular area at $2000 \mathrm{~Hz}$ is plotted in Fig. 11, and the interpretation of the decomposition result is shown in Fig. 12. The acoustic part was concentrated on a disk centered at $\left(k_{x}, k_{y}\right)=(0,0)$ with a radius of $2 \pi f / c$, where $f$ was the frequency $(2000 \mathrm{~Hz})$ and $c$ was the speed of sound $(340 \mathrm{~m} / \mathrm{s})$. The turbulent part was located on a vertical region located at $k_{x}=2 \pi f /\|\vec{a}\|$, where $\vec{a}$ was the wind velocity vector on the window $(120 \mathrm{~km} / \mathrm{h}$ in this case).

The sound pressure level (SPL) at each frequency was generally computed by integrating the component on a disk enclosing the acoustic locus. The hydrodynamic contribution was then calculated by summing all the remaining values after the acoustic component had been removed. This operation can be done for each frequency (see Fig. 13). As predicted, the turbulence pressure was the main component of the total pressure, 


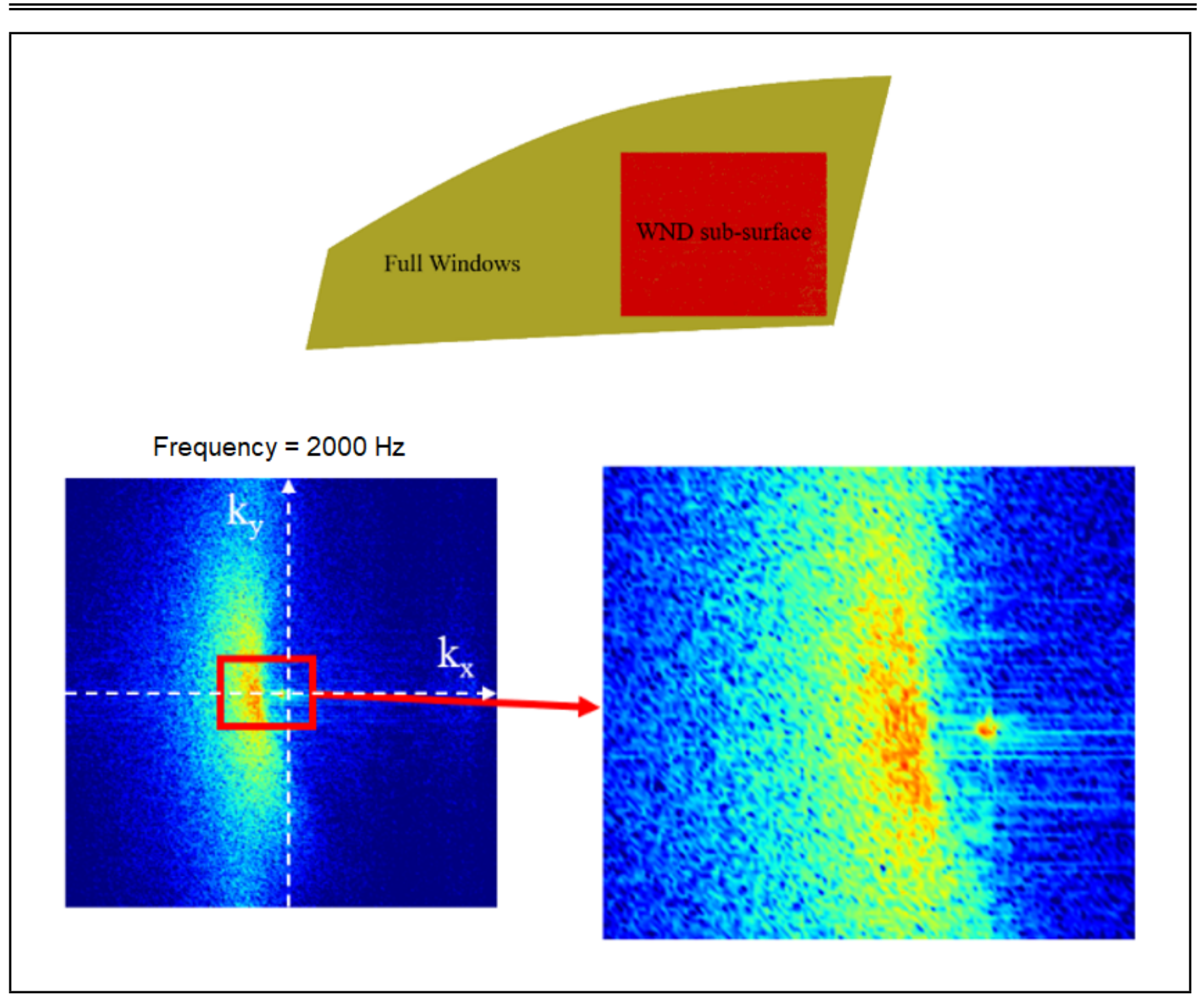

Figure 11. Wave number decomposition at $2000 \mathrm{~Hz}$.

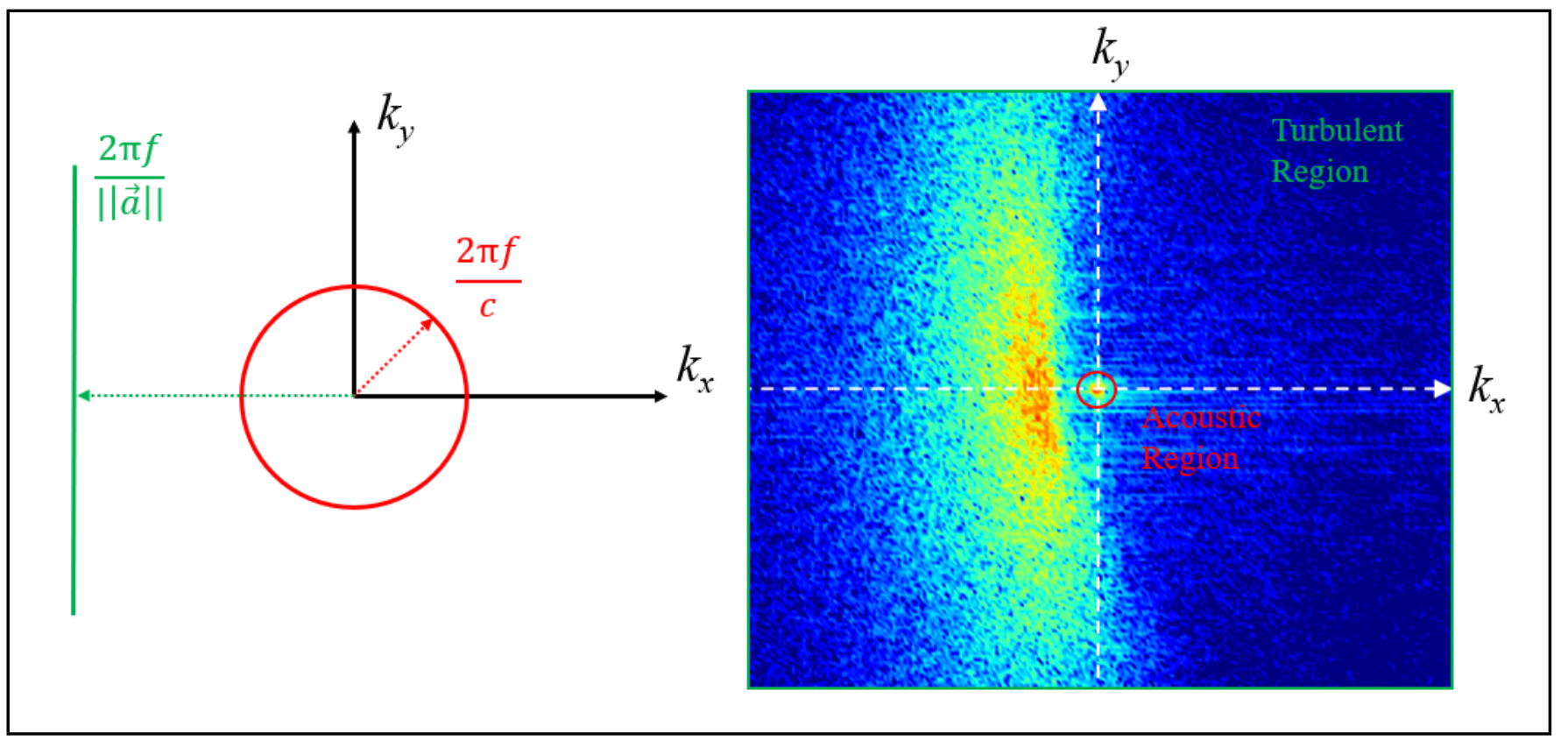

Figure 12. Locus identification for wave number decomposition. 


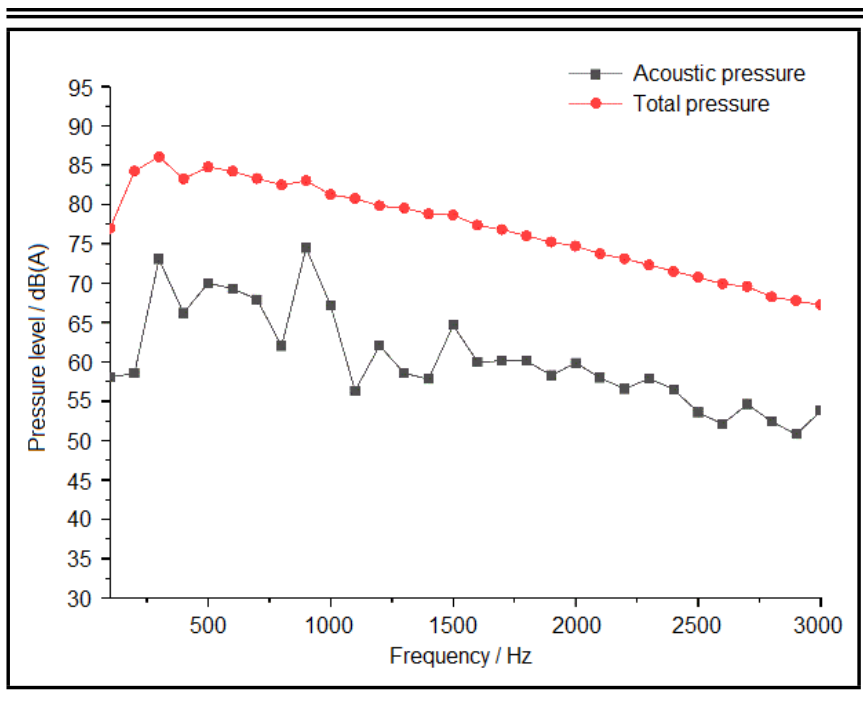

Figure 13. Acoustic and total contribution of wall pressure fluctuations.

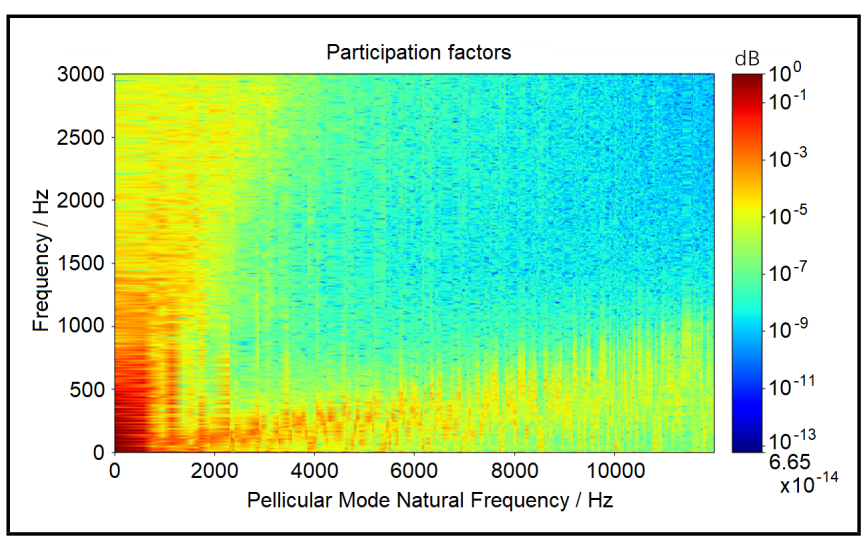

Figure 14. Pellicular mode decomposition plot.

which was nearly $20 \mathrm{~dB}(\mathrm{~A})$ greater than acoustic pressure.

PMD was also a commonly used method to decompose the pressure. Compared with WND, the advantage of PMD was that it can decompose the pressure on irregular surfaces. Similar with WND, the surface pressure on the left window had been decomposed. The PMD pressure participation factored (illustrated in formula(6)) on the left window at different pellicular mode frequencies is plotted in Fig. 14.

Based on the definition of the participation factors, the two pressure contributions were located at two specific positions as illustrated in Fig. 15. The acoustic component was integrated over a red triangular area which enclosed the acoustic region. All the participation factors, which were outside of the acoustic region, were integrated to build the turbulent part.

The comparison between the WND and PMD at the same rectangular area is illustrated in Fig. 16. It shows that the acoustic pressure was almost the same, while the total pressure obtained by PMD decreased rapidly after $500 \mathrm{~Hz}$. This situation may be attributed to the insufficient number of pellicular modes.

Figure 17 showed the comparison between acoustic contributions by different methods and regions. The acoustic pressure on sub-surface obtained by WND and PMD was almost the same, while the acoustic pressure of the full window obtained by PMD was slightly different with the former.
Table 5. Interior sound velocity considering reverberation time.

\begin{tabular}{|c|c|c|}
\hline Frequency & Real part & Imaginary part \\
\hline 100 & 340 & 53.42688 \\
630 & 340 & 19.78766 \\
800 & 340 & 15.58278 \\
1000 & 340 & 12.46623 \\
1250 & 340 & 9.97298 \\
1600 & 340 & 7.791391 \\
2000 & 340 & 6.233113 \\
2500 & 340 & 4.98649 \\
3150 & 340 & 3.957532 \\
\hline
\end{tabular}

Table 6. Glass parameters.

\begin{tabular}{|c|c|}
\hline Young's modulus $(\mathrm{GPa})$ & 70.2 \\
Poisson's ratio & 0.23 \\
Density $\left(\mathrm{kg} / \mathrm{m}^{3}\right)$ & 2500 \\
Thickness $(\mathrm{mm})$ & 5 \\
\hline
\end{tabular}

Table 7. PVB parameters

\begin{tabular}{||c|c|}
\hline Young's modulus (MPa) & 1.4 \\
Poisson's ratio & 0.47 \\
Density $\left(\mathrm{g} / \mathrm{cm}^{3}\right)$ & 1.07 \\
Thickness $(\mathrm{mm})$ & 0.76 \\
\hline
\end{tabular}

\section{INTERIOR NOISE CALCULATION}

ACTRAN was also used to calculate the interior wind noise. Before the interior noise calculation, a vibro-acoustic model should be established. The size of the grid should be satisfied with the criterion that each wavelength should contain at least 6 elements. As the maximum calculating frequency was $3000 \mathrm{~Hz}$, the maximum grid size was set as $18 \mathrm{~mm}$, as shown in Fig. 18.

In addition, it was necessary to consider the attenuation of the sound when it propagated in the internal cavity. Since the materials of cavity were complex, the sound absorption boundary was set by adding an imaginary part to the sound velocity, which was calculated based on the reverberation time. The air velocity applied to the interior cavity is shown in Table 5. Furthermore, the window glass parameters needed to be defined in advance. All the windows had a PVB (polyvinyl butyral) interlayer, and the required parameters are shown in Table 6 and Table 7.

Otherwise, two monitoring points were set up near the driver's ears, as shown in Fig. 19.

The next step was to get the transfer function. A relatively small set of modes (200-300 modes) and the orthogonality property of sub-space was used to load the vibro-acoustic model and determine the transfer function of each pellicular mode shape in the frequency range of interest.

Like the participation factor, the visualization of the transfer functions is illustrated in Fig. 20. The $x$-axis is the pellicular mode frequency, the $y$-axis is the frequency of interest.

Two straight lines could be noted in the transfer function map: one is the dashed white line; another is the solid white line. The transfer function was higher around the dashed white line because the acoustic wavelengths on the excitation side were consistent with the acoustic wavelengths in the car cavity. The solid white line whose slope was 0.098 represented the turbulent locus, and the transfer function was very low below this line because of the low transfer efficiency of turbulent pressure. 


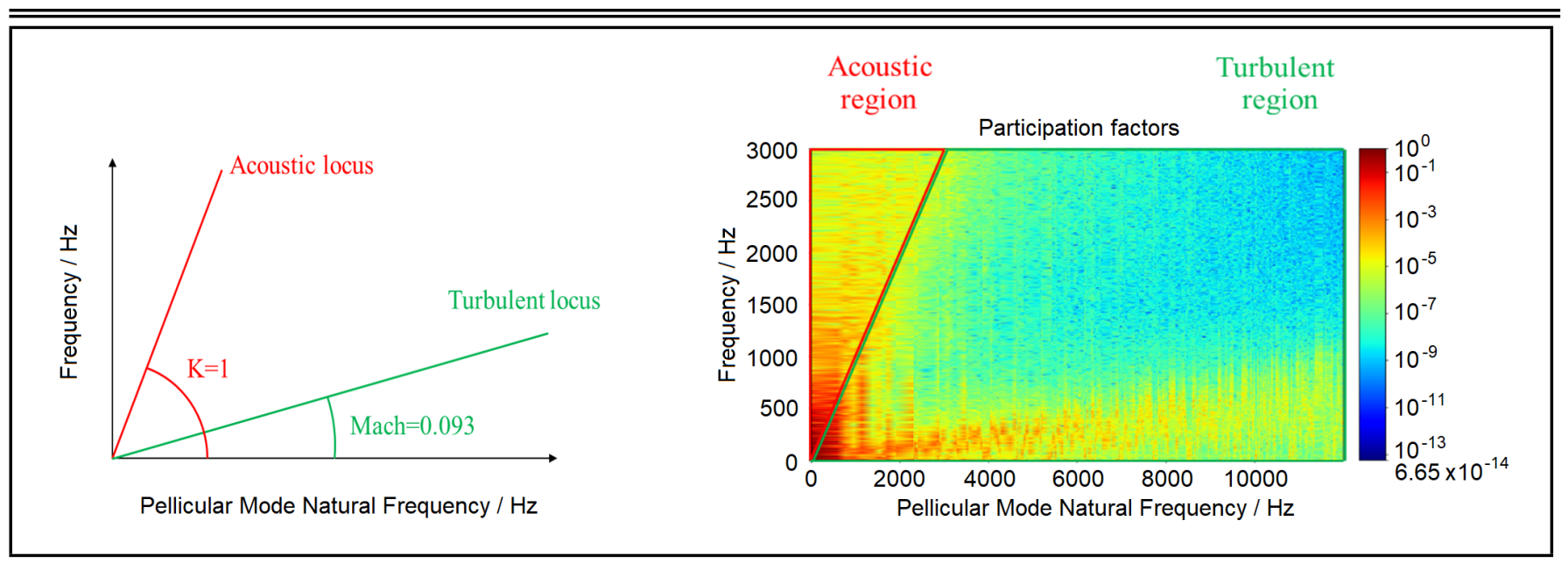

Figure 15. Locus identification for pellicular mode decomposition.

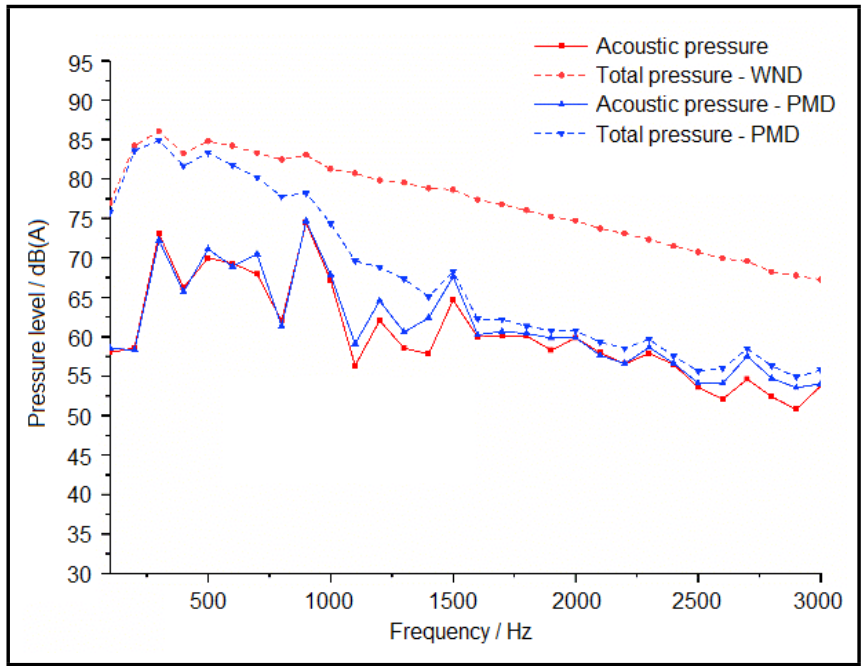

Figure 16. Acoustic pressure and total pressure on the sub-surface.

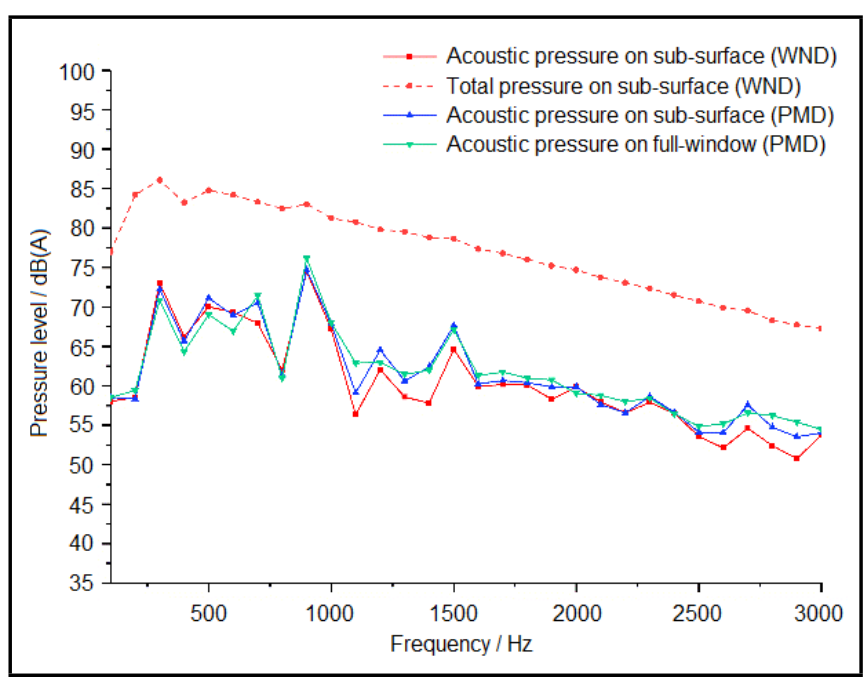

Figure 17. Acoustic contributions comparison between methods.

There were still some small contributions between the acoustic and turbulent wavelength. Even if these contributions had small amplitude, the transfer efficiency to the cavity could be very high when the wavelengths of contributions matched the bending wavelength of the structure, as illustrated in Fig. 21.

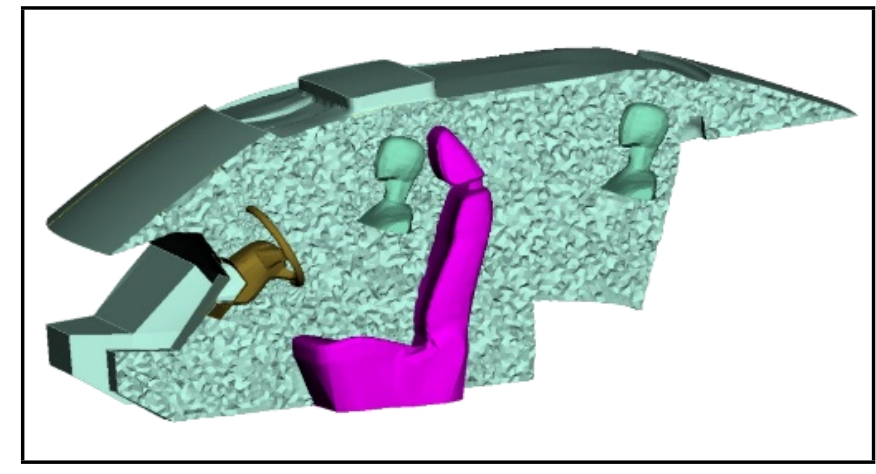

Figure 18. The vibro-acoustic model.

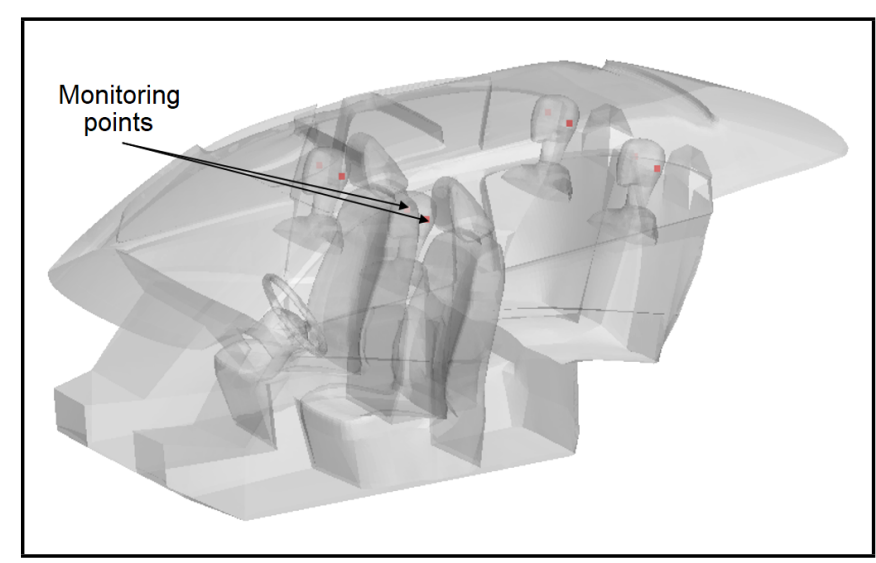

Figure 19. Monitoring points near the driver's ears.

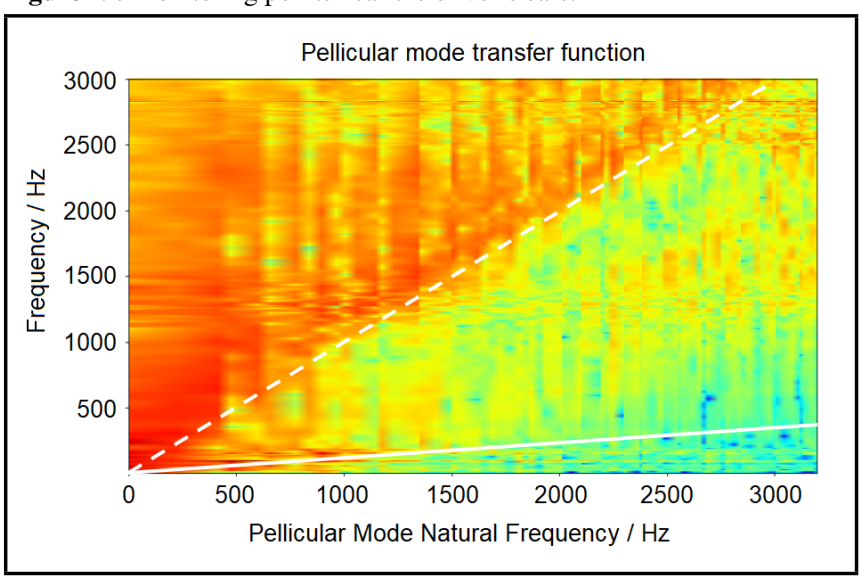

Figure 20. Pellicular modes transfer functions for interior acoustic pressure. 


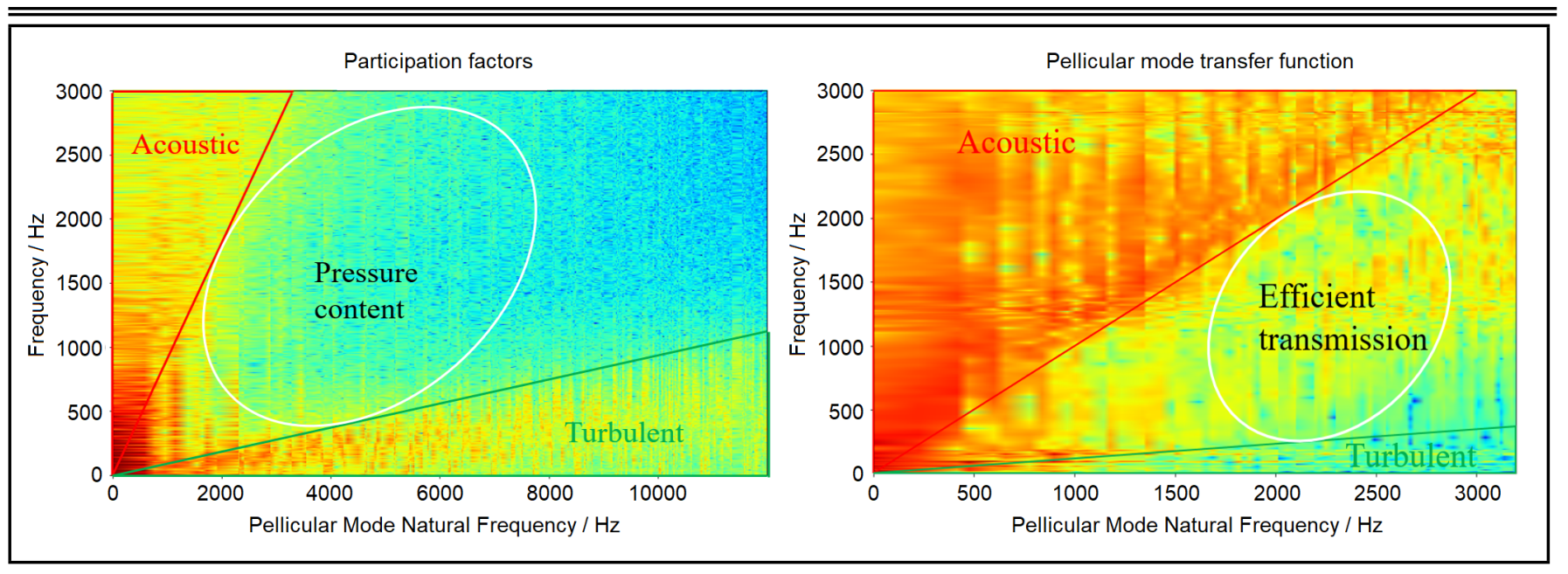

Figure 21. Pressure content located between acoustic and turbulent locus has efficient transmission.

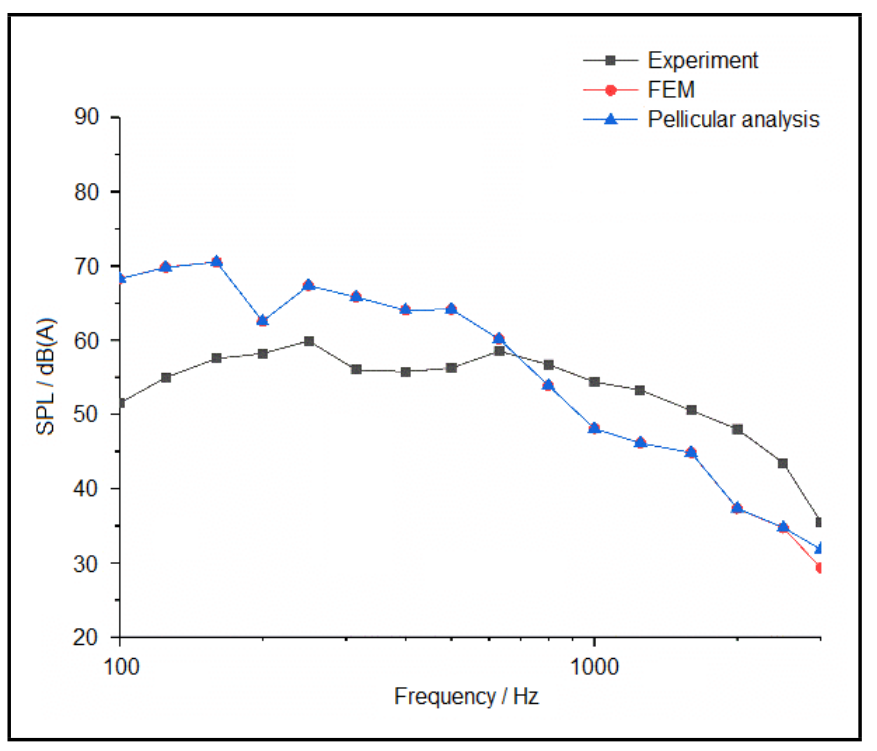

Figure 22. SPL in driver's left ear.

Table 8. Calculation resource.

\begin{tabular}{|c|c|c||}
\hline & FEM & Pellicular analysis \\
\hline Memory & $128 \mathrm{~GB}$ & $128 \mathrm{~GB}$ \\
The first calculation time & $20 \mathrm{~h}$ & $20 \mathrm{~h}$ \\
The second calculation time & $20 \mathrm{~h}$ & $0.1 \mathrm{~h}$ \\
\hline
\end{tabular}

Once the transfer functions had been obtained, the participation factors of the pellicular pressure decomposition was used to get the final interior sound pressure. After getting the transfer function, the computation time to calculate the interior noise was quite short.

The calculation results of the $1 / 3$ octave SPL of the driver's left ear obtained by FEM and pellicular analysis are plotted in Fig. 22. Furthermore, FEM was used to do the calculation twice, and so was pellicular analysis. The comparison of the computational cost is also listed in Table 8.

In the first calculation, the calculation time of the FEM and pellicular analysis was basically the same, but in the second calculation, pellicular analysis had an obvious advantage in calculation time since the transfer function had been obtained, and the results obtained by the two methods were almost the same. Therefore, it is more efficient to use a pellicular analysis to calculate the noise inside the vehicle.
Both the results obtained by the FEM and pellicular analysis had some error with the experimental results. The comparisons revealed that the computational result was over-prediction in the low-frequency and under-prediction in the high-frequency. The overall sound pressure level (OSPL) error was about $8 \mathrm{~dB}(\mathrm{~A})$. The reason for the error may be as follows: 1) The constraint of real vehicle window glasses is very complicated, and it was simplified in the simulation model. 2) Test error of acoustic attenuation data in the cavity. 3) Due to the convenience of changing the rearview mirror during the experiment, the sound insulation characteristic of the triangle area of the rearview mirror was poor. 4) The structure of real interior cavity is complicated, and the simulation model was difficult to be completely consistent with it. For the above reasons, there was an error between the calculated transfer function and the actual transfer function, which lead to errors in the calculation of the SPL in the vehicle.

However, the FPL on the window surface monitoring points is in good agreement with the experimental values, and the pressure decomposition results are in line with the theory, which shows that the calculation process is rigorous. In the future work, we will do further comparative research on the calculation of the SPL in the cavity.

\section{CONCLUSIONS}

In this paper, WND and PMD are used to decompose the surface pressure on the windows, and pellicular analysis is used to further calculate the interior noise. The two methods can successfully decompose the pressure on window surface into turbulent and acoustic pressure, and the contribution of turbulence is greater than that of acoustics, which is consistent with the aeroacoustics theory. Compared with FEM, the calculation time after the transfer function obtained by pellicular analysis can be shortened by more than $99 \%$ when calculating the interior noise, and the results are almost the same as FEM. Although there are some errors in the SPL results and experiments in the cavity, the whole calculation process is rigorous. Therefore, calculate interior wind noise by pellicular analysis may be a better choice for the early development process of a car whose appearance is often changed. 


\section{ACKNOWLEDGEMENT}

The research was supported by the Open fund of State Key Laboratory of Comprehensive Technology on Automobile Vibration and Noise \& Safety control (Grant No. 1803J-W65GNZX-2018-0215). The authors also would like to thank the MSC Software for the help in using ACTRAN during this work.

\section{REFERENCES}

1 Cabrol, M., Detandt, Y., Hartmann, M., and Mutzke, A. A comparison between the effects of turbulent and acoustic wall pressure fluctuations inside a car, Proc. of 18th AIAA/CEAS Aeroacoustic Conference, (2012). https://dx.doi.org/10.2514/6.2012-2202

2 Vanherpe, F., Baresh, D., Lafon, P., and Bordji, M. Wavenumber-frequency analysis of the wall pressure fluctuations in the wake of a car side mirror, Proc. of 17th AIAA/CEAS Aeroacoustics Conference, (2011). https://dx.doi.org/10.2514/6.2011-2936

3 Bremner, P. and Wilby, J. Aero-vibro-acoustics: Problem statement and methods for simulation-based design solution, Proc. of 8th AIAA/CEAS Aeroacoustics Conference \& Exhibit, (2002). https://dx.doi.org/10.2514/6.2002-2551

4 Arguillat, B., Ricot, D., Bailly, C., and Robert, G. Measured wavenumber: Frequency spectrum associated with acoustic and aerodynamic wall pressure fluctuations, The Journal of the Acoustical Society of America, 128 (4), 1647-1655, (2010). https://dx.doi.org/10.1121/1.3478780

5 Haxter, S. and Spehr, C. Infinite beamforming: wavenumber decomposition of surface pressure fluctuations, Proc. of the 5th Berlin Beamforming Conference, (2014).

6 Wang, X., Mizushiri, K., and Yokoyama, H., et al. Wavenumber-frequency spectrum analysis of pressure fields around an automobile, Proc. of the ASME-JSMEKSME 2019 8th Joint Fluids Engineering Conference, Volume 3A: Fluid Applications and Systems, (2019). https://dx.doi.org/10.1115/AJKFluids2019-4806

7 Ganty, B., Copiello, D., Detandt, Y., and Jeong, C. A method to identify the acoustic contribution in pressure fluctuations acting on a realistic car's side window, Proc. of 44th INTER-NOISE and NOISE-CON Congress and Conference, (2015).
8 Jacqmot, J., Detandt, Y., Lielens, G., and Copiello, D. Vibro-aero-acoustic simulation of side mirror wind noise and strategies to evaluate pressure contributions, Proc. of ISMA2016 including USD2016, (2016).

9 Bremner, P. G. and Zhu, M. Recent progress using SEA and CFD to predict interior wind noise, SAE Technical Paper, (2003). https://dx.doi.org/10.4271/2003-01-1705

10 Oberai, A. A., Roknaldin, F., and Hughes, T. J. R. Computational procedures for determining structural-acoustic response due to hydrodynamic sources, Computer Methods in Applied Mechanics \& Engineering, 190 (3), 345-361, (2000). https://dx.doi.org/10.1016/S0045-7825(00)002061

11 Kurosawa, Y. Predicting automotive interior noise including wind noise by Statistical Energy Analysis, International Journal of Mechanical and Mechatronics Engineering, 10 (3), 635-641, (2016). https://dx.doi.org/10.5281/zenodo.1132056

12 Schell, A. and Cotoni, V. Prediction of interior noise in a sedan due to exterior flow, SAE International Journal of Passenger Cars-Mechanical Systems, 8 (3), 1090-1096, (2015). https://dx.doi.org/10.4271/2015-01-2331

13 Cho, M., Kim, H. G., Oh, C., Ih, K. D., Khondge, A., Mendonca, F., Lim, J., Choi, E.-S., Ganty, B., and Hallez, R. Benchmark study of numerical solvers for the prediction of interior noise transmission excited by A-pillar vortex, Proc. of 43rd Inter-noise \& Noise-con Congress \& Conference, 3139-3937, (2014).

14 Wang, Y. S., Sui, L. N., Yin, Z. Y., Wang, X. L., Liu, N. N., and Guo, H. A hybrid prediction for wind buffeting noises of vehicle rear window based on LES-LAA method, Applied Mathematical Modelling, 47, 160-173, (2017). https://dx.doi.org/10.1016/j.apm.2017.03.018

15 Actran 19 User's Guide: Free Field Technologies SA. (2018). 